\title{
Soliton complexes in dissipative systems: Vibrating, shaking, and mixed soliton pairs
}

\author{
J. M. Soto-Crespo \\ Instituto de Óptica, CSIC, Serrano 121, 28006 Madrid, Spain \\ Ph. Grelu \\ Laboratoire de Physique, de l'Université de Bourgogne, UMR CNRS 5027, Faculté des Sciences Mirande, \\ Avenue Savary, Boîte Postale 47870, 21078 Dijon Cedex, France \\ N. Akhmediev and N. Devine \\ Optical Sciences Group, Research School of Physical Sciences and Engineering, The Australian National University, \\ Canberra Australian Capital Territory 0200, Australia \\ (Received 17 November 2006; published 25 January 2007)
}

\begin{abstract}
We show, numerically, that coupled soliton pairs in nonlinear dissipative systems modeled by the cubicquintic complex Ginzburg-Landau equation can exist in various forms. They can be stationary, or they can pulsate periodically, quasiperiodically, or chaotically, as is the case for single solitons. In particular, we have found various types of vibrating and shaking soliton pairs. Each type is stable in the sense that a given bound state exists in the same form indefinitely. New solutions appear at special values of the equation parameters, thus bifurcating from stationary pairs. We also report the finding of mixed soliton pairs, formed by two different types of single solitons. We present regions of existence of the pair solutions and corresponding bifurcation diagrams.
\end{abstract}

DOI: 10.1103/PhysRevE.75.016613

PACS number(s): 42.65.Tg, 47.20.Ky

\section{INTRODUCTION}

Pulse-pulse interaction is one of the main issues in the design of soliton-based optical transmission lines [1,2]. The way one bit of information interacts with another can destroy or protect the system under consideration. Since the generalized use of the optical amplifier, long-haul, high-bit-rate transmission lines have now become all optical [3]. A longhaul all-optical system is basically a dissipative one, i.e., all losses in the system are compensated for through the interaction with an external pump [4]. Thus, if we want to take advantage of soliton-based transmission, we need to know the features of solitons and their interactions in dissipative systems at a fundamental level.

Robust soliton pairs do exist in dissipative systems. Since their stable existence was first predicted [5] in optical systems governed by the complex Ginzburg-Landau equation, they have been experimentally observed on various occasions in fiber lasers [6]. The fact that dissipative solitons, when they exist, usually have a fixed profile, allows us to describe the interaction between two of them using just two variables, namely the separation $\rho$ and the phase difference $\theta$ between the two pulses. Therefore the dynamics of a pair of solitons can be described in a two-dimensional phase space that is usually called the "interaction plane" [5]. In many cases, the dynamics of the interaction between two solitons is simple enough so that its analysis can be done without ambiguity in a reduced two-dimensional phase space. However, this is not always the case, as our present study shows.

Even single solitons in dissipative systems can have complicated behavior. They can be pulsating, creeping, or exploding [7] and exhibit many other types of dynamics [8]. These are all determined by the parameters of the system. Being equipped with this knowledge, one would expect that soliton pairs could also show complicated behaviors. In par- ticular, we have found that a soliton pair can pulsate or evolve chaotically. Systems with an infinite number of degrees of freedom are likely to exhibit a wide range of complicated dynamics, and the reduction to simpler systems with a two-dimensional phase space cannot be applied as a general rule. At least for some regions of the parameter space, more degrees of freedom need to be considered to understand the dynamics. In these cases, the interaction plane is clearly not adequate to describe the dynamics of pairs in its full complexity.

In this paper, we consider such complicated cases when soliton pairs are oscillating in time, either periodically or chaotically. In each case, the soliton pair exists indefinitely in time as a bounded, localized two-soliton solution, thus manifesting stability. We have found three types of bound states; we call them the vibrating soliton pair (VSP), shaking soliton pair (SSP), and mixed soliton pair. The VSP shows simple oscillations of the soliton pair variables. These oscillations can be considered as limit cycles of our dynamical system with an infinite number of degrees of freedom (see discussions on this subject in Chapter 1 in [9]). The SSP is essentially a strange attractor. Its behavior is somewhat similar to that of a single exploding soliton [7]. We have also found transitions between these various propagation regimes which occur when the parameters of the system are changed, and these are manifest as bifurcations in the soliton pair dynamics. An interesting feature of this complex dynamics is that it is specific for the soliton pair: each soliton forming the pair has perfectly stable stationary behavior, when isolated, for the same set of the equation parameters.

\section{MASTER EQUATION}

We are dealing, in this paper, with a dynamical system governed by the cubic-quintic complex Ginzburg-Landau 
equation (CGLE) [10], which in optics has been widely used to describe the pulsed operation of passively mode-locked lasers. The CGLE is

$i \psi_{z}+\frac{D}{2} \psi_{t t}+|\psi|^{2} \psi+\nu|\psi|^{4} \psi=i \delta \psi+i \epsilon|\psi|^{2} \psi+i \beta \psi_{t t}+i \mu|\psi|^{4} \psi$

When used to describe passively mode-locked lasers, the CGLE represents a distributed propagation model, in which $z$ is the distance traveled inside the cavity, $t$ is the retarded time, $\psi$ is the normalized envelope of the field, $D$ is the group velocity dispersion coefficient, with $D= \pm 1$, depending on whether the group velocity dispersion (GVD) is anomalous or normal, respectively, $\delta$ is the linear gain-loss coefficient, $i \beta \psi_{t t}$ accounts for spectral filtering or linear parabolic gain $(\beta>0), \epsilon|\psi|^{2} \psi$ represents the nonlinear gain (which arises, e.g., from saturable absorption), and the term with $\mu$ represents, if negative, the saturation of the nonlinear gain, while the one with $\nu$ corresponds, also if negative, to the saturation of the nonlinear refractive index. During numerical computations with the propagation equation, the magnitude that we most often monitor is the energy $Q$ carried by a certain solution after a propagated distance $z$. It is defined by

$$
Q=\int_{-\infty}^{\infty}|\psi(t, z)|^{2} d t
$$

When $Q$ oscillates on propagation, we will denote its maximum and minimum by $Q_{M}$ and $Q_{m}$, respectively.

Chaotic soliton pairs were first found by Turaev, Vladimirov, and Zelik [11]. However, the authors of [11] used an equation that differs from (1) by an additional term responsible for a weak signal injected into the laser, and the main reason for chaotic motion in their work was this additional perturbation, i.e., they induced a chaotic component in the two-soliton solution artificially. Our results here are obtained with Eq. (1) without any additional terms. This means that chaotic or shaking soliton pairs can exist in the dissipative system without any external perturbations. The existence of these additional solutions can be considered as an essential characteristic of the dissipative system, rather than a feature induced by additional forces. The main way to find them is to correctly choose the parameters of the system. Once the parameters are chosen, the system will consistently produce the solution in the form of a vibrating or chaotic bound state of two solitons.

Before entering this subject deeply, we consider the problem of the excitation of bound states. First, we should mention that, in order to generate a two-soliton solution in numerical simulations, we have to choose the initial condition correctly. In our previous work [5], we used two single solitons, found from preliminary numerical simulations, added a finite phase difference between them, and located them at a fixed separation from each other [see Eq. (7) of [5]]. If the bound state does exist, this initial condition converges to it, provided that the phase difference and the separation are chosen within certain limits. In many cases, a broader class of localized initial conditions can also be used. There is no
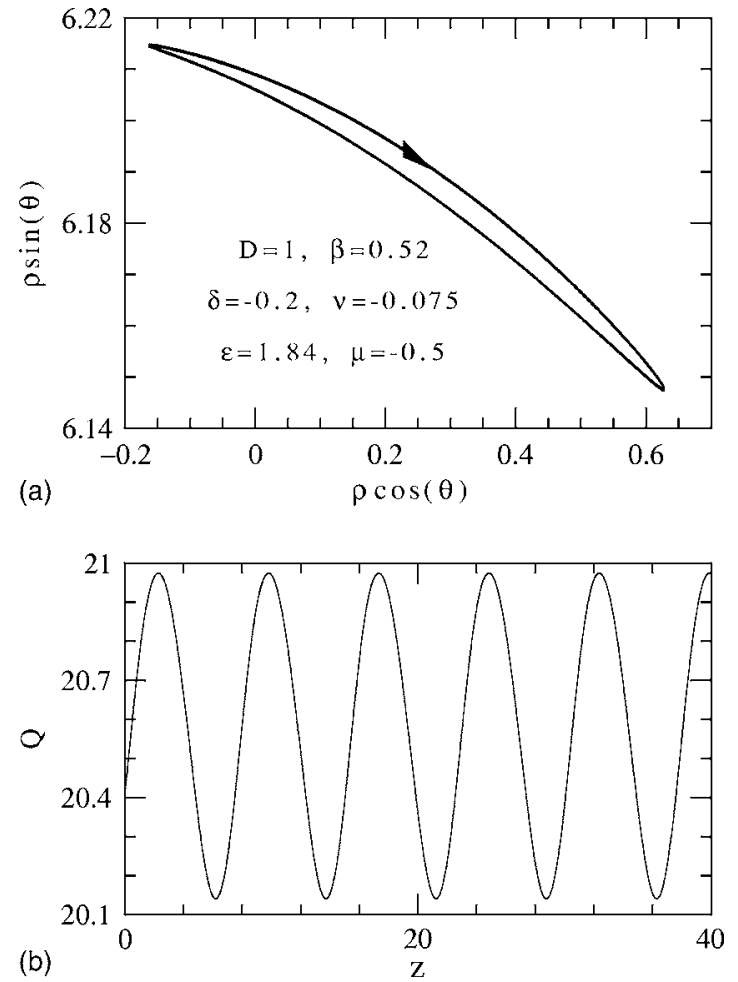

FIG. 1. (a) Interaction plane limit cycle that corresponds to purely periodic oscillations between the two solitons. The arrow indicates the clockwise rotation of the trajectory. (b) Periodic evolution of the energy $Q$ for the same case. The equation parameters used in the simulation are written in (a).

certainty that a stable soliton pair will be excited. However, when it is excited, the solution converges to the same bound state if it is the only pair that exists for a given set of parameters. Each bound state has a basin of attraction that is large enough to allow us to generate these solutions with a certain facility. When two types of solution exist simultaneously, each of them has its own basin of attraction.

\section{VIBRATING SOLITON PAIRS}

In Ref. [5], we found stable soliton pairs with $\pm \pi / 2$ phase difference between the two pulses. These two equivalent pairs can be represented by two points in the interaction plane. Trajectories that start at nearby points in this plane converge to the fixed point, which can be considered as a stable focus of the dynamical system. On changing the parameters of the system, we can find those values for which its stability becomes marginal and the fixed point is transformed into a center, thus allowing periodic orbits around the center. These periodic orbits are marginally stable. They cannot be considered as attractors of a dynamical system. In the present work, we have found a different type of periodic orbit. These orbits are stable robust formations which are limit cycles of the CGLE. As such, they are attractors of the nonlinear dynamical system. An example is shown in Fig. 1(a).

Let us suppose that we use an initial condition in the form of two solitons separated by a finite distance. When starting from an arbitrary point in the interaction plane, located in- 


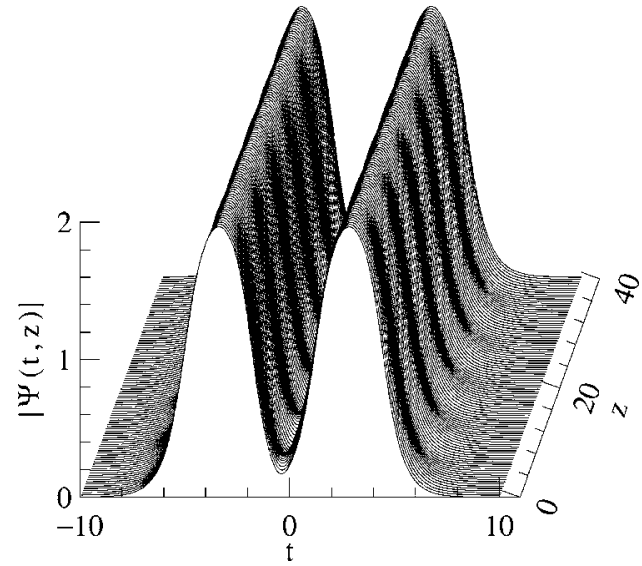

FIG. 2. Evolution of the pulse profile for the vibrating soliton pair (VSP). Parameters of the simulation are the same as in Fig. 1.

side or outside of the limit cycle in Fig. 1(a), the trajectory converges to this limit cycle, rather than to a fixed point. Moreover, when the initial conditions are not exactly two solitons at a fixed distance, but two pulses with a shape that only approximately resembles solitons, the trajectory also converges to the same limit cycle. The early part of the trajectory in the phase space depends on the initial condition and can be rather complicated. Thus, all such parts of the trajectories have been removed from Fig. 1(a) for the sake of clarity. Only the limit cycle, which is the final part of stable evolution, is shown. It is represented by the solid curve in the figure. The arrow shows the direction of motion in the interaction plane. We call this type of solution a vibrating soliton pair [12].

An interesting observation is that, for some given sets of the system parameters, only two types of solution exist: single stationary solitons and vibrating pairs. Soliton pairs with fixed distance and phase difference do not exist for this set of parameters, or at least we have not observed them. Thus, any initial condition which is a bound pair of two pulses located in the basin of attraction will cause creation of a VSP, rather than of a stationary soliton pair.

The trajectory shown in Fig. 1 is noticeably asymmetric relative to the vertical line which corresponds to a phase difference of $\pi / 2$. The distance $\rho$ between the two maxima of the pulses oscillates, as does the phase difference. This set of two pulses has a finite velocity, moving toward the right (positive $z$ direction). The peak amplitude of the pulse on the right-hand side is slightly larger than that of the pulse on the left. This asymmetry comes from the nonsymmetric phase relationship between the two pulses. However, due to the $t \leftrightarrow-t$ symmetry of the CGLE, there always exists its symmetric VSP solution moving toward the left and having a phase difference close to $-\pi / 2$. The periodic evolution of the energy $Q$ vs $z$ is shown in Fig. 1(b). This single periodic curve is very close to being harmonic.

The pulse profile evolution is shown in Fig. 2 for the same propagation distance as in Fig. 1(b). The periodic evolution is better observed from the slopes of the two solitons rather than at the maxima. The reason that the vibration appears with a small amplitude in this diagram is mainly due to the fact that, in the example chosen, it is mostly the relative

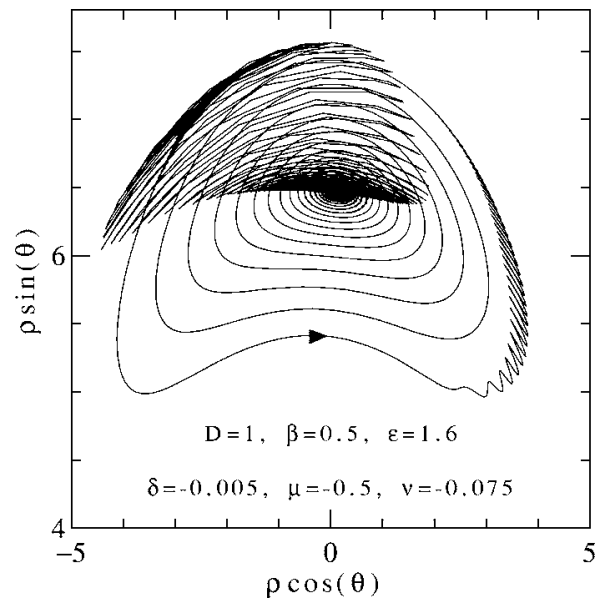

FIG. 3. Trajectory of the motion on the interaction plane for a soliton pair that is spontaneously shaken. The approach to the center and departure from it follow very different paths on the plane. Parameters of the simulation are written inside the figure.

phase which oscillates, while the relative separation oscillates only by $0.5 \%$, as can be seen in Fig. 1(a). This solution can be considered as the pulsating two-soliton generalization of a single pulsating soliton [7,8]. However, pulsations here are solely due to the interaction between the two solitons. Single pulsating solitons do not exist at the set of parameters chosen for these simulations. At the same time, two single pulsating solitons do not create a VSP. The pulsations of both solitons causes them to merge into one. Thus, we can consider a VSP as a new object in the family of localized solutions of the CGLE.

\section{SHAKING SOLITON PAIRS}

A second object that we have found numerically is what we call a shaking soliton pair. Its dynamics demonstrates the presence of chaotic effects in the evolution of soliton pairs. These are stationary pairs that have an intrinsic instability of an oscillatory type. The pair can be represented on the interaction plane as a fixed point which is an unstable-stable focus. An example of such a point is shown on the interaction plane in Fig. 3. The trajectory that describes the evolution of this pair is a spiral that winds out off the focus, makes a loop, and winds back to the initial point, thus repeating the cycle again and again. The cycles are similar to each other but are not exactly the same. For clarity, only one of the cycles is shown in Fig. 3. In a global evolution, each cycle is a homoclinic orbit returning back to the same point. The process of return is clearly seen in Fig. 3.

The center manifold of this dynamics is at least four dimensional. The inward (outward) spiraling trajectory can be related to a fixed point with its corresponding linearized stability analysis providing two complex conjugate eigenvalues with negative (positive) real part. Thus, a complete description needs at least two pairs of complex eigenvalues. The trajectory in this reduced phase space escapes the fixed point in one two-dimensional subspace and returns to this point in another two-dimensional subspace. 


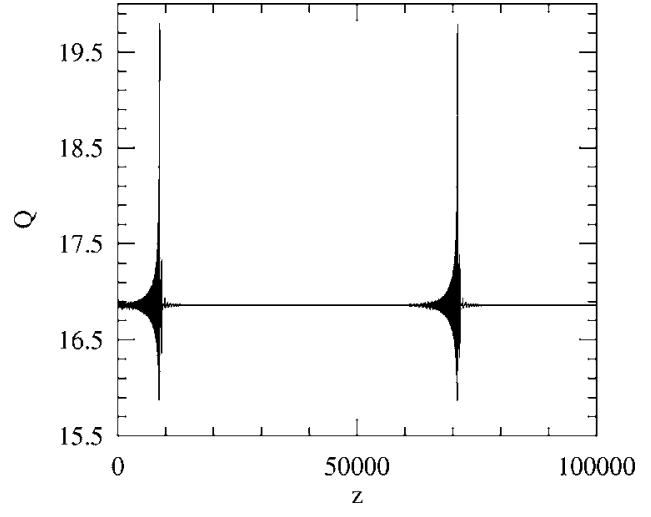

FIG. 4. Energy $Q$ versus $z$ for a soliton pair that is spontaneously shaken as an instability takes place. It corresponds to the same case as the one shown in Fig. 3.

The evolution of the total energy $Q$ of the shaking pair is shown in Fig. 4. When the pair is in the nearly stationary part of the evolution, the energy appears to be constant. This corresponds to the fixed point in Fig. 3. In this example, the soliton pair spends most of the propagation time very close to this fixed point. On the other hand, when the pair is disturbed by the instability, the energy changes and evolves with the oscillations. Two shaking parts of the evolution are clearly seen in Fig. 4. After the instability is over, the energy returns to the same constant value as before. The cycles repeat indefinitely in ways that are similar, but not exactly the same. The evolution of the pulse profile during one cycle is illustrated in Fig. 5.

If we change the parameters of the system, the stationary part of the evolution may become shorter in comparison with the shaking part. To demonstrate this, we significantly changed the parameters $\epsilon, \beta$, and $\delta$. The resulting plot for the energy $Q$ versus $z$ is shown in Fig. 6. Despite the shorter stationary part of the trajectory, the shaking feature appears, again and again, almost periodically.

We stress that the orbit does not repeat itself at each of the shaking parts of the evolution, thus confirming the fact that many frequencies are involved in this dynamics. In fact, when the values of the equation parameters are slightly changed, the differences between the cycles can be made

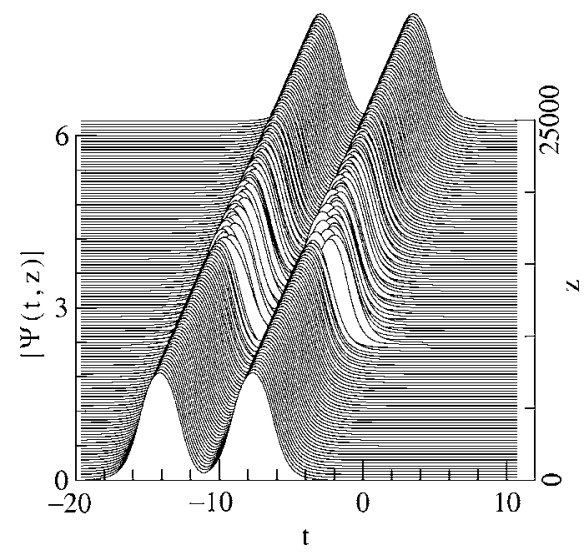

FIG. 5. Evolution of the pulse profile of the shaking soliton pair shown in Fig. 4.

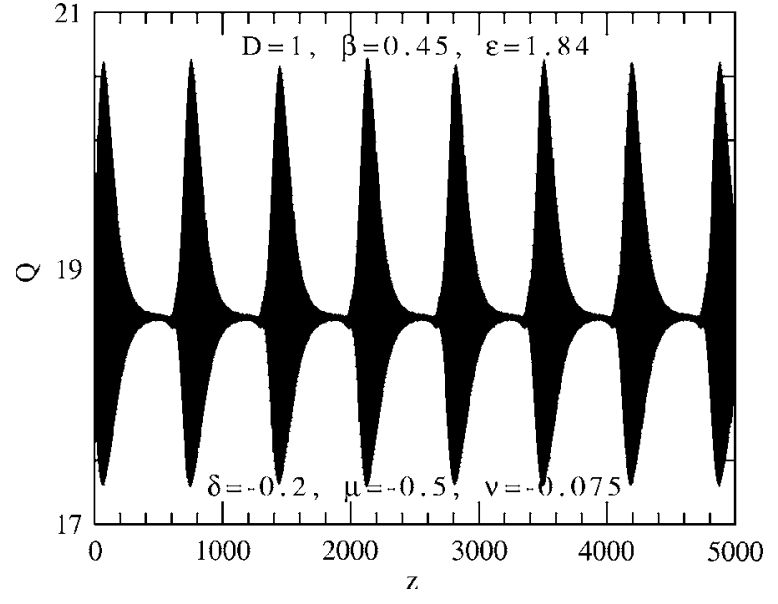

FIG. 6. Another example of a spontaneously shaken soliton pair. The parameters of the simulation are written inside the figure. This example shows that the shaking feature can occur for a relatively wide range of the system parameters.

considerably larger, showing that the chaotic nature of the motion becomes more pronounced.

The peak amplitudes $A_{1}$ and $A_{2}$ of the two solitons in the pair for one cycle of evolution are shown in Fig. 7. The two amplitudes have almost the same value at the nearly stationary part of the evolution. They start to oscillate due to the instability, but the amplitudes of oscillation are clearly different. When the instability is over, the oscillations decay and the soliton pair becomes nearly stationary again. The soliton pair is slightly asymmetric in that the right-hand side (RHS) pulse (gray dotted line) has an average amplitude which is larger than that of the pulse on the left (solid line). This asymmetry also comes from the phase asymmetry, as previously discussed.

The phase trajectory on the interaction plane for the same dynamics of the two pulses is shown in Fig. 8. The counterclockwise direction of the trajectory is indicated by the arrow. The right-hand side part of the orbit corresponds to the part in Figs. 7 (6) where the amplitude (energy) experiences larger variations, while the left-hand side trajectory corresponds to having the amplitude (energy) almost constant. In

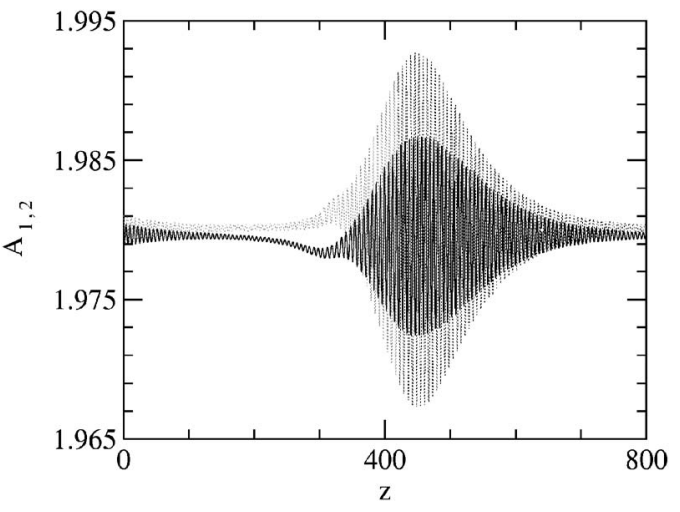

FIG. 7. Peak amplitudes of the two solitons forming the shaking pair. Gray dotted line is for $A_{1}$, and black solid line is for $A_{2}$. Only about one cycle of the spontaneous shaking represented in Fig. 6 is shown here, with a higher resolution along the $z$ direction. 


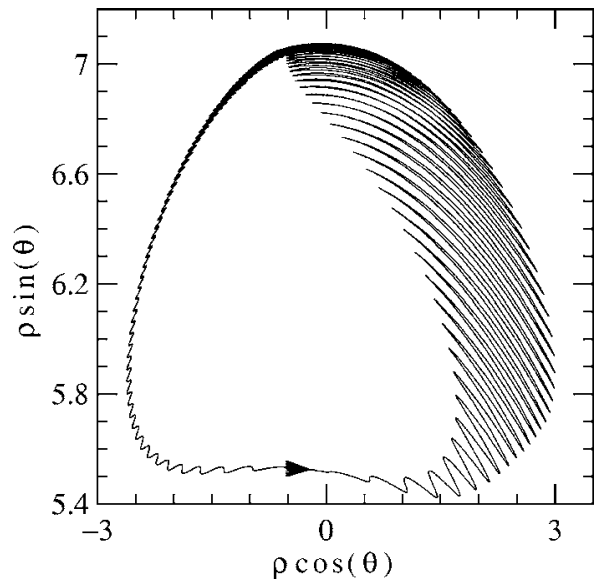

FIG. 8. Trajectory on the interaction plane showing one cycle of shaking in Fig. 6. The direction of the evolution is indicated by the arrow. There is no particular fixed point for this example. For $2 / 3$ of the time, the system stays on the left-hand side part of the orbit, bursting into higher amplitude oscillations on the right-hand side of the loop.

the right-hand part, the motion consists mainly of fast oscillations of the relative phase, superimposed on a slower stretching of the soliton pair separation.

The trajectory in Fig. 8 is shown only for a single burst of instability in Fig. 6. To be specific, $z$ varies from 0 to 800 . Plotting the next loop of the trajectory shows a similar pattern; however, the phase of the oscillations is shifted relative to the first loop. Plotting several loops would show the chaotic variation of the phase evolution.

The same set of parameters admits a second type of pair solution, thus revealing bistability. The energy $Q$ versus $z$ for this solution is shown in Fig. 9(a). This plot shows the "period tripling" phenomenon for the "envelope" of fast oscillations. This conjecture is confirmed by plotting the trajectory for this motion on the interaction plane. The latter is shown in Fig. 9(b). One can see three loops in the trajectory. Two of them almost overlap, while the third one, having a smaller amplitude of oscillation, is located inside them. The trajectory is roughly "repeated" after the third loop, but with chaotic fluctuations, showing that the motion is a complicated mix of regular and chaotic features.

The examples above show nontrivial dynamics of soliton interactions in dissipative systems. The type of dynamics is defined solely by the parameters of the system. Some sets of parameters produce a particular behavior, while others admit two or more types. In order to know what to expect in the dynamics, we have to classify the solutions in terms of the various system parameters.

\section{RANGE OF EXISTENCE AND BIFURCATIONS}

Each type of pair described above exists in a certain region of the parameter space. Finding complete sets of regions of existence for various types of soliton pairs is a tedious task. The first essential step would be to find a region of existence for stable stationary single solitons. To some extent, this work has been carried out in our previous papers
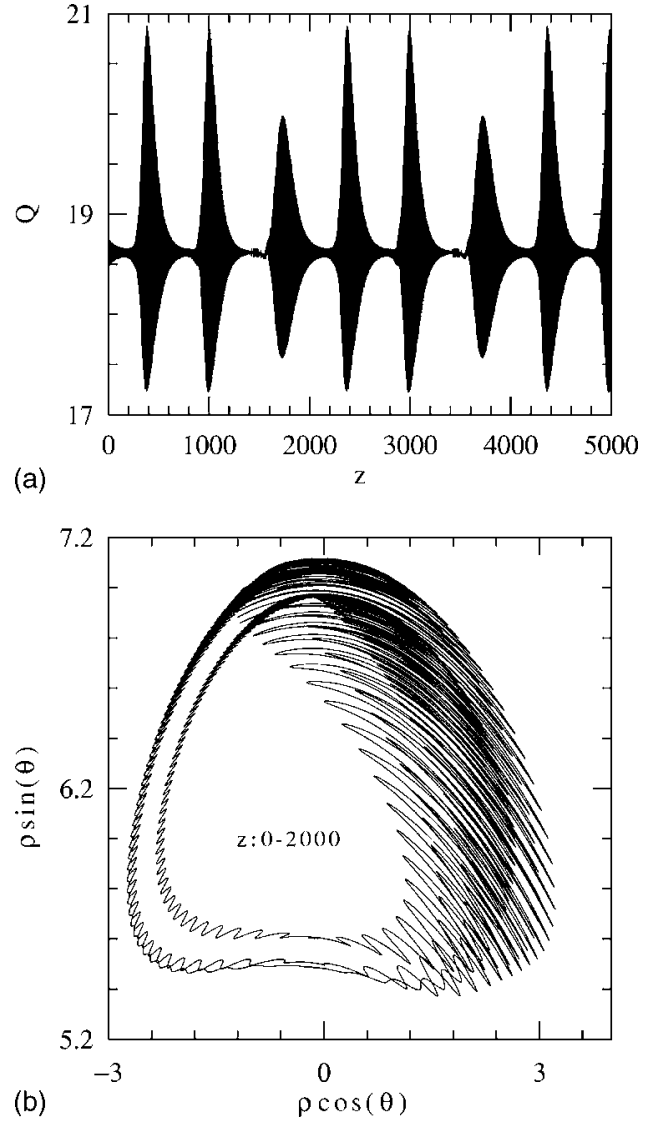

FIG. 9. (a) Energy $Q$ versus $z$ for the alternative evolution of the system with the same set of parameters as in Fig. 6. Period tripling of the envelope of oscillations can clearly be seen. (b) Trajectory in the interaction plane for the same simulation.

[15]. Inside the region, we can look for stationary soliton pairs, and this is still a relatively easy and short task. Once a stable stationary pair is found for a single set of parameters, we change the values of the parameters, and try to delimit the areas where the specific pair exists. Usually, only two parameters are changed, and the rest are kept constant. This procedure allows us to make a two-dimensional graphical representation of the regions, while keeping the computational burden within reasonable limits. The soliton pairs may gain qualitatively new features at the edges of these regions. These features usually appear in the form of bifurcations.

In order to find the bifurcations, we further simplified the technique. In particular, we monitored the energy of the pair while changing only one parameter of the system. When the soliton pair is stationary, the energy has a fixed value for a given set of soliton parameters. This changes when the parameters are changed, but it reaches a constant value for each new point in the parameter space. If the soliton pair starts pulsating at a bifurcation point, then the energy becomes a periodic function of $z$. Strict periodicity appears after convergence to the new solution. After convergence to periodic or quasiperiodic motion had occurred, we monitored all the local minimal and maximal values of the energy.

In most of the simulations, we fixed all the parameters except the nonlinear gain coefficient $\epsilon$. An example of a plot of maxima $Q_{M}$ and minima $Q_{m}$ of the energy $Q$ versus $\epsilon$, for 


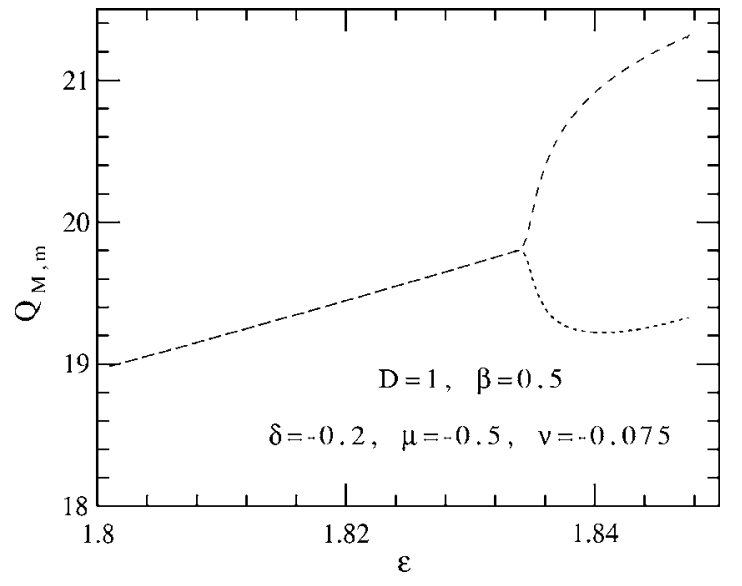

FIG. 10. Bifurcation diagram obtained by plotting the maximal and minimal values of oscillating $Q$ from the $Q$ vs $\epsilon$ values observed as the soliton pair evolves along the $z$ variable. This diagram clearly shows the transition from stationary soliton pairs to vibrating ones. The bifurcation occurs at $\epsilon=1.835$. The parameters of the system are written inside the figure.

the case when a stationary pair is transformed into a simple pulsating pair, is shown in Fig. 10. The energy $Q$ takes a stationary value when $\epsilon$ is within the range [1.05, 1.834], although the figure shows it in the much smaller interval $[1.8,1.834]$. At $\epsilon=1.835$, the bifurcation occurs and the energy starts to oscillate between the lower and upper curves in Fig. 10. This bifurcation is from a stable fixed point to a limit cycle (Hopf bifurcation). The soliton pair starts to vibrate at this point. The energy varies from the lower value to the upper one when the representative point moves along the limit cycle in Fig. 1.

Another type of bifurcation is shown in Fig. 11. Relative to Fig. 10, only the value of $\beta$ has been changed, from 0.5 to 0.45 , but the dynamics changes dramatically. In fact, the diagram in Fig. 11 shows a stationary value of energy $Q$ when $\epsilon$ changes in the interval $[1.82,1.829]$. Bifurcation takes place at $\epsilon=1.829$. The soliton pair starts to shake, thus causing the energy to evolve chaotically. In contrast to the previous case, there is no single frequency and no fixed value for the am-

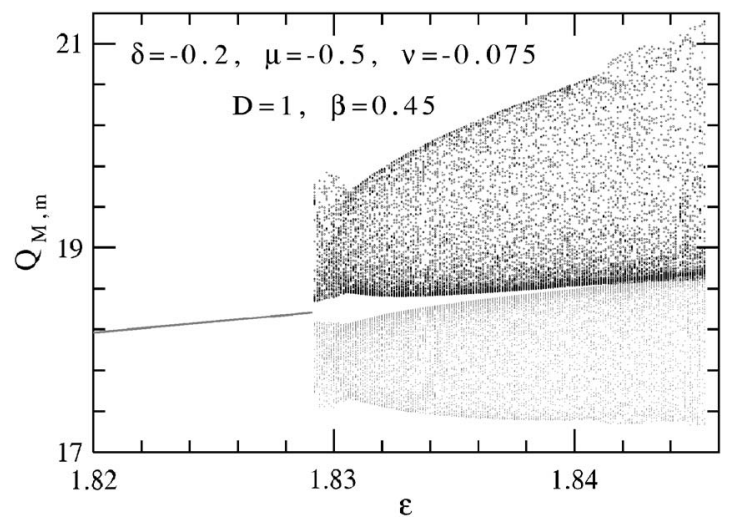

FIG. 11. Bifurcation diagram constructed in the same way as the one in Fig. 10. This plot reveals a transition from stationary soliton pairs to shaking soliton pairs at $\epsilon=1.829$. Parameters of the simulation are shown inside the figure.

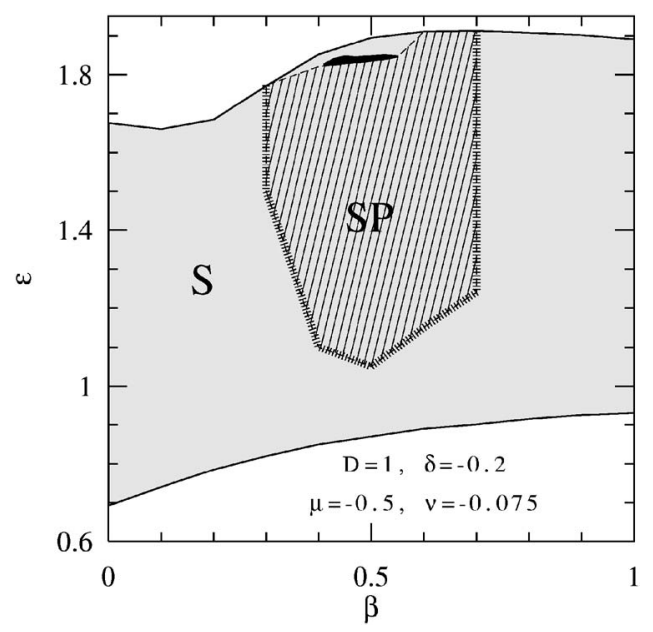

FIG. 12. Regions of existence of single stable solitons (S) and various forms of soliton pairs-(SPs). The dashed region corresponds to stable stationary bound states. The black region above SP is the one with stable VSPs and SSPs.

plitude of oscillations. Plotting every local maximum and minimum in the changes of energy creates the lower and upper ranges of what appears as an energy band in Fig. 11. The plot shows that, at the point $\epsilon=1.829$, the stable stationary pair is transformed into a shaking pair with maximum and minimum values of energy evolving like those in Fig. 4. The transition that is observed in this case is abrupt, thus allowing us to classify the bifurcation that is occurring in Fig. 11 as subcritical. We have found two solutions that exist simultaneously in the small region above the point $\epsilon=1.829$. Increasing or decreasing $\epsilon$ allows us to demonstrate each type of solution.

Now we turn our attention to the regions of existence of various types of solutions. An accurate mapping in the parameter space requires high accuracy and fine parameter scanning in the simulations. Diagrams which are similar to those shown above have to be constructed when the second parameter changes in the simulations. The results of such a mapping are shown in Fig. 12. In fact, the largest region, indicated in gray in this plot, is the zone in the parameter space where stable single solitons (S) exist. We expect stable soliton pairs to appear only inside this region. The smaller hatched region corresponds to stable stationary soliton pairs (SP). Generally, as Fig. 12 shows, stable soliton pairs exist over a relatively large range of parameters. The value of $\epsilon$ can vary from $\approx 1.1$ to $\approx 1.8$ and $\beta$ from $\approx 0.3$ to $\approx 0.7$. Thus, these two parameters can almost double their values and soliton pairs still remain stable.

VSPs and SSPs in our simulations appear in a much smaller region above the hatched area. This region is shown in black. The two types of pairs, VSPs and SSPs, do not coexist with stationary pairs. Thus, the region for VSPs and SSPs, marked in black, has a distinct boundary with the hatched area shown in Fig. 12. The small size of the black region indicates that the types of soliton pairs found in this work are far from being typical cases. Only careful adjustment of the parameters allows us to generate them. It may happen that additional perturbations, similar to those consid- 


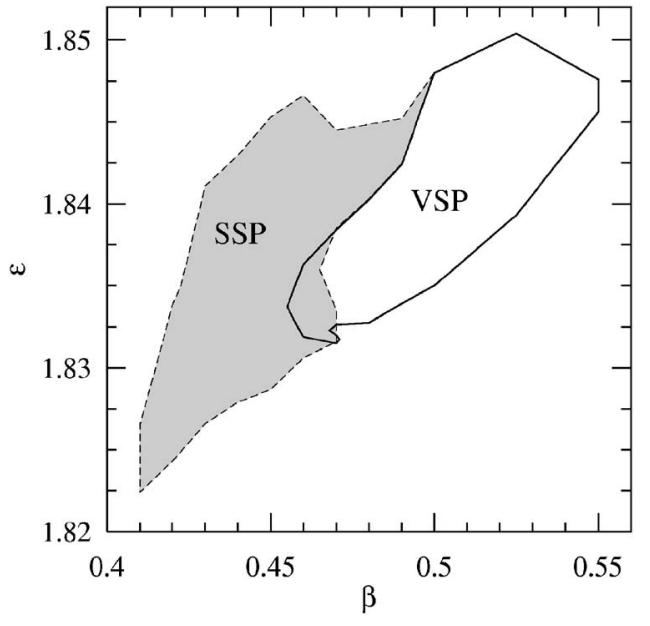

FIG. 13. The regions of existence of SSPs and VSPs in the $(\beta, \epsilon)$ plane. This is a magnification of the small region shown in Fig. 12 in black. Higher resolution of this region allows us to separate it into two subregions for SSPs and VSPs. The overlapping part of the two regions indicates that the two types of soliton pairs can coexist.

ered in [11], may expand the region of their existence.

The regions for VSP and SSP solutions are shown separately, with higher resolution, in Fig. 13. VSPs appear in the white region surrounded by the solid black curve, while the region for SSPs is shown shaded in gray. For each type of pair, the size of the region of existence is very similar. The lower boundaries are the lines of bifurcation from the stationary soliton pairs. This magnification also clearly shows a region of overlap between the two regions. Two types of soliton pairs coexist in this region, and each one is stable. The initial conditions determine which one is excited in any particular simulation. In order to demonstrate this, we fixed the parameter $\beta=0.47$, and changed $\epsilon$, step by step, using the previous solution as the initial condition for the simulation at the new $\epsilon$.

The bifurcation diagram for soliton pairs constructed in this way is shown in Fig. 14. The gray dots are calculated for the parameter $\epsilon$ increasing in simulations. Specifically, we start at $\epsilon=1.83$ and find a stationary pair at this point. We take this solution as the initial condition and find the next soliton pair at $\epsilon=\epsilon+\Delta \epsilon$, with $\Delta \epsilon=+0.0001$. The black points are obtained when we decrease $\epsilon$ using the same increment with a minus sign. When the CGLE admits only one soliton pair that is stationary and stable, the simulations result in the same solution, independent of the direction of changing $\epsilon$. On the other hand, the two solutions differ just above the bifurcation point, indicating the presence of bistability. Above the region of bistability, there is only one stable VSP solution, so that the two branches again coincide in the form of upper and lower values of energy for the vibrating solution.

The plot in Fig. 14 shows the second bifurcation from VSP to SSP at around $\epsilon \approx 1.8385$. The maximal and minimal values of the oscillating energy now split into upper and lower bands, indicating chaotic motion. A new frequency appears in the dynamics at this point, thus making the overall motion rather complicated.

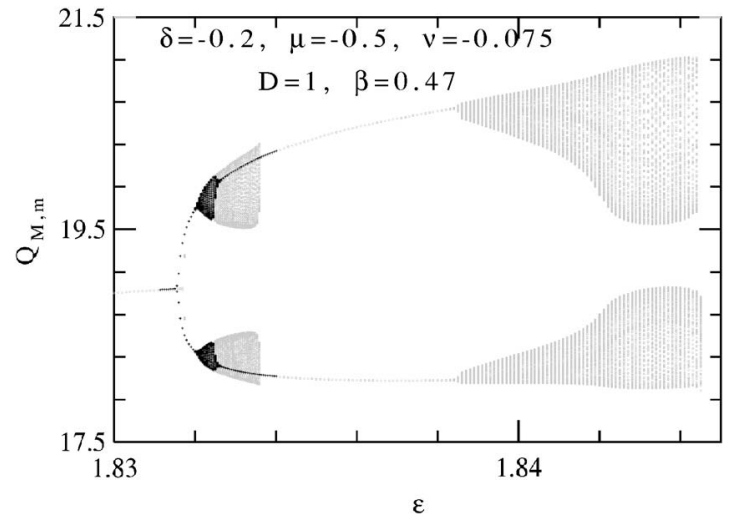

FIG. 14. Bifurcation diagram obtained by increasing (gray) and decreasing (black) values of $\epsilon$. This plot explicitly shows the coexistence of two types of soliton pair in the interval $1.832<\epsilon$ $<1.8336$. This interval corresponds to the overlapping region for SSPs and VSPs in Fig. 13.

\section{MIXED PAIRS}

All the results in the previous sections are related to soliton pairs that consist of two identical plain pulses. It is known that soliton pairs may involve more complicated composite pulses [13]. However, it was not known that the CGLE admits mixed soliton pairs when one of the solitons in the pair is a plain one and the second one is a composite soliton [14]. Clearly, this has to be considered as a very special case. First, to obtain a mixed pair, the parameter set has to be chosen in a region where each type of soliton is stable. The latter is relatively small, as we know from previous studies [15]. Second, the propagation constants for the two different solitons are different. Thus, the phase difference between the two solitons cannot be kept constant. Any such solution would rotate around the origin when represented by a point on the interaction plane. Consequently, it is hard to imagine that mixed pairs would exist at all. Despite these issues, we were able to observe stable mixed pairs, and one example is shown in Fig. 15.

Due to the constantly increasing phase difference between the two solitons, a mixed pair creates a periodic motion more

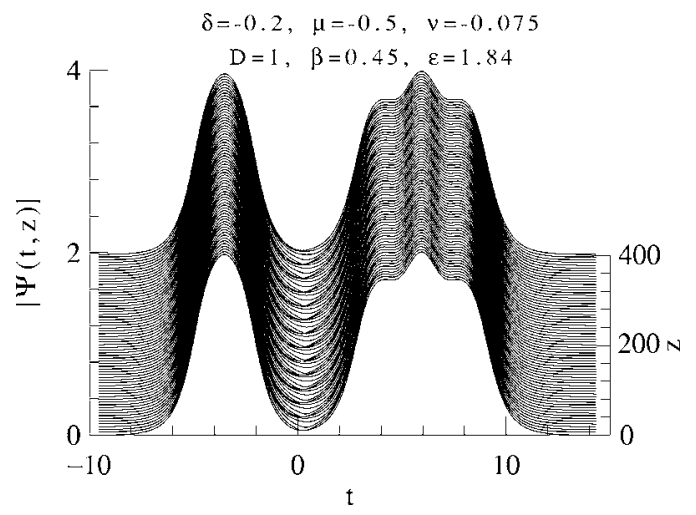

FIG. 15. Stable bound state of a plain soliton and a composite soliton, which we call a mixed soliton pair. Its small vibration is hardly visible in the evolution plot. 


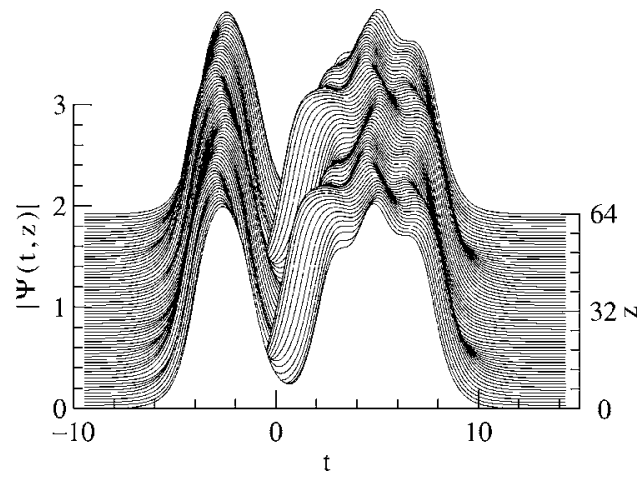

FIG. 16. One period of oscillation of a mixed soliton pair. Parameters of the simulation are shown in Fig. 17.

naturally than a pair consisting of two equivalent solitons. Moreover, this is the reason why a mixed soliton pair can never be stationary. Figure 15 shows the oscillatory dynamics that occurs predominantly in the low-amplitude region between the two solitons. In general, rotation of the relative phase implies periodic attraction and repulsion of the two pulses, thus creating periodic evolution. We can say that any mixed pair is originally a VSP.

In most cases, the oscillations can be noticed only between solitons as in Fig. 15, since the interaction between the two pulses is weak. Then the oscillation frequency between the two solitons is the beat frequency defined by the difference between the propagation constants of the individual solitons. The oscillations of energy for these solutions would also be small. However, changing the parameters of the system allows us to find mixed pairs with oscillations that are more pronounced than those in Fig. 15. One such case is shown in Fig. 16.

A composite pulse is more susceptible to a change of its shape, since it, in turn, consists of two fronts bound to the central bright soliton. The front that is closer to the plain pulse on the left moves with a higher amplitude than the other one. Only one full period of oscillations of the mixed pair is shown in Fig. 16. The period, in this case, is equal to four periods of beating between the two individual solitons. Thus, this solution is the result of two period doublings in the sequence of period-doubling bifurcations (see below). As the size of the composite soliton changes appreciably, the energy of the mixed pair oscillates with a relatively high amplitude. These oscillations are shown in Fig. 17. The oscillations in energy are quite far from being purely harmonic, thus confirming that these vibrations are not a simple beating between the two solitons. Indeed, they are the result of a sequence of two period-doubling bifurcations (see below).

A mixed pair is subjected to transformations when the equation parameters are changed, and, in order to follow these, we can construct a bifurcation diagram in the same way as for that for the pair of two plain solitons. Thus, we monitored the energy $Q$ of the pair when changing the parameter $\epsilon$. As the solution is always vibrating and the energy is never stationary, there are at least two extremal values of the energy for any value of the parameter $\epsilon$. The minimal and maximal values of $Q$ for this solution are shown in Fig. 18 for $\epsilon$ in the interval $[1.81,1.86]$. The remaining parameters were kept constant.

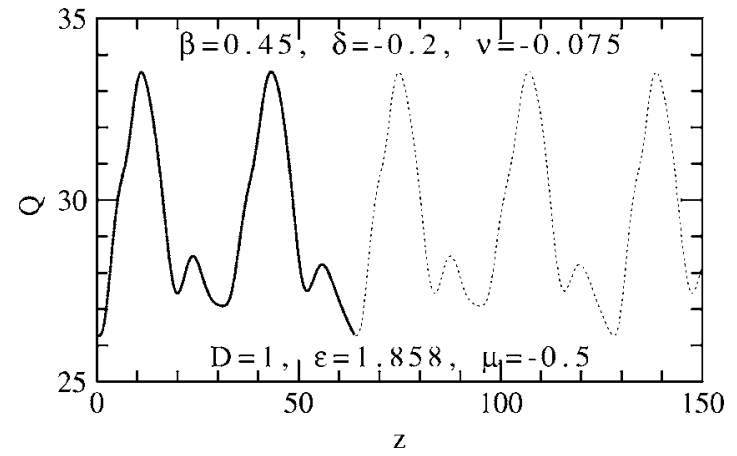

FIG. 17. Periodic evolution of energy of a mixed pair. The part of the curve corresponding to the period presented in Fig. 16 is shown by the solid line.

For small values of $\epsilon$ in the given interval, the two solitons interact weakly. Consequently, oscillations of energy are very small, and the minima and maxima of $Q$ are close to each other. The maxima and minima almost coincide at $\epsilon$ $=1.81$. Increasing $\epsilon$ results in stronger oscillations, so that the two values become well separated at around $\epsilon=1.85$. When $\epsilon=1.855$, a bifurcation occurs and the solution starts to pulsate with a frequency that is twice the beat frequency. At this point, we need to plot all local minima and maxima of the energy. Consequently, the two branches of energy in Fig. 18 split into four. A further increase in $\epsilon$ results in a sequence of period-doubling bifurcations and chaotic motion of the pair. This can be clearly seen in the inset of Fig. 18, which represents the same bifurcation diagram with higher resolution. Neither multifrequency dynamics nor chaotic motion destroys the soliton pair. It remains a stable localized object up to $\epsilon=1.86$. The pair only develops instability above this limiting value. The two pulses then annihilate each other, creating just a single composite soliton.

Our further studies have shown that pairs formed by two composite pulses [13] can also evolve in a complicated way, just like pairs formed by two plain pulses. These pairs can be

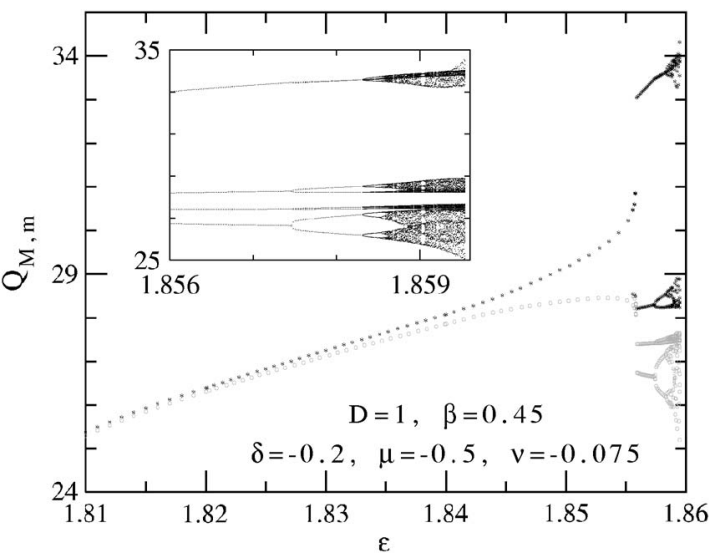

FIG. 18. Bifurcation diagram showing changes in the behavior of the mixed soliton pair illustrated in Figs. 15 and 16. The gray and black dots correspond to the minimal and the maximal values of the energy of the oscillations. Multiple bifurcations, including period doubling, occur on the RHS part of the diagram. This part is shown in greater detail in the inset of this plot. 
vibrating, shaking, and generally chaotic. Transformations between these forms occur as bifurcations which are similar to those given in the present work. The results of these simulations are of interest in themselves and will be presented elsewhere.

\section{CONCLUSIONS}

In this work, we have studied three types of soliton pair in dissipative systems governed by the complex GinzburgLandau equation. The interaction of solitons in dissipative systems appears to be more complicated than we previously thought. There are subtle effects in the interaction that lead to the vibrating behavior of pairs. These vibrations can be almost harmonic or have a chaotic component, similar to intermittency in low-dimensional systems. We call these bound states vibrating or shaking pairs, depending on the number of frequencies involved in the dynamics. Another observation that we presented in this work is that the two solitons in the bound state do not have to be identical. The interaction of two different types of solitons produces naturally vibrating pairs, and even shaking pairs. This complicated dynamics of bound states can appear without any additional external perturbations. A correct choice of system parameters is the only requirement for the appearance of these solutions.

These observations can have far-reaching consequences. Single solitons can be perfectly stable for a given set of parameters. However, this does not mean that a bound state formed from them is either stationary or stable. Moreover, their relations can be highly complicated. Such is the life of dissipative solitons.

\section{ACKNOWLEDGMENTS}

The work of J.M.S.C. was supported by the MCyT under Contracts No. BFM2003-00427 and No. FIS2006-03376, the work of Ph.G. is supported by Agence Nationale de la Recherche, and N.A. acknowledges support from the Australian Research Council. The authors are grateful to Dr. Ankiewicz for a critical reading of the manuscript.
[1] F. M. Mitschke and L. F. Mollenauer, Opt. Lett. 12, 355 (1987).

[2] C. Desem and P. L. Chu, IEE Proc.-J: Optoelectron. 134, 145 (1987).

[3] A. Hasegawa and M. Matsumoto, Optical Solitons in Fibers, 3rd ed. (Springer-Verlag, Berlin, 2003), Chap. 6.

[4] Z. Bakonyi, D. Michaelis, U. Peschel, G. Onishchukov, and F. Lederer, J. Opt. Soc. Am. B 19, 487 (2002).

[5] N. Akhmediev, A. Ankiewicz, and J. M. Soto-Crespo, Phys. Rev. Lett. 79, 4047 (1997).

[6] Ph. Grelu, F. Belhache, F. Gutty, and J. M. Soto-Crespo, Opt. Lett. 27, 966 (2002).

[7] J. M. Soto-Crespo, N. Akhmediev, and A. Ankiewicz, Phys. Rev. Lett. 85, 2937 (2000).

[8] N. Akhmediev, J. M. Soto-Crespo, and G. Town, Phys. Rev. E
63, 056602 (2001).

[9] Dissipative Solitons, edited by N. Akhmediev and A. Ankiewicz (Springer, Heidelberg, 2005).

[10] I. S. Aranson and L. Kramer, Rev. Mod. Phys. 74, 99 (2002).

[11] D. Turaev, A. Vladimirov, and S. Zelik, Weierstrass Institute fur Angewandte Analysis and Stochastik, Berlin, Report No 1152, 2006 (unpublished).

[12] M. Grapinet and Ph. Grelu, Opt. Lett. 31, 2115 (2006).

[13] N. Akhmediev, A. S. Rodrigues, and G. Town, Opt. Commun. 187, 419 (2001).

[14] V. V. Afanasjev, N. Akhmediev, and J. M. Soto-Crespo, Phys. Rev. E 53, 1931 (1996).

[15] N. N. Akhmediev and A. Ankiewicz, Solitons, Nonlinear Pulses and Beams (Chapman and Hall, London, 1997), Chap. 13. 